\title{
Relationship Between Lymph Node Metastasis and Lymph Node Density and Preoperative Neutrophil-Lymphocyte Ratio in Patients Undergoing Radical Cystectomy
}

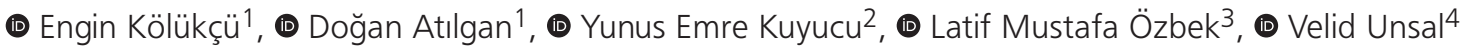 \\ ${ }^{1}$ Gaziosmanpaşa University Faculty of Medicine, Department of Urology, Tokat, Turkey \\ ${ }^{2}$ Gaziosmanpaşa University Faculty of Medicine, Department of Biostatistics, Tokat, Turkey \\ 3 Private Atasam Hospital, Clinic of Urology, Samsun, Turkey \\ ${ }^{4}$ Mardin Artuklu University, Faculty of Health Sciences and Central Research Laboratory, Mardin, Turkey
}

\begin{abstract}
Objective: This study aimed to evaluate the relationship between the preoperative neutrophil-lymphocyte ratio (NLR) and lymph node metastasis and lymph node density after radical cystectomy in patients with invasive urinary bladder cancer

Materials and Methods: Data of 89 patients who underwent radical cystectomy were examined. Our study included only cases with stage 2 urothelial bladder cancer. They were classified according to the lymph node status based on the surgical specimen. Patients with negative results were classified as group 1 and those with positive results as group 2. Patients in group 2 were further evaluated in two subgroups according to their lymph node density. Accordingly, group 2A consisted of patients with lymph node density of $<20 \%$, and group $2 B$ involved those with lymph node density of $\geq 20 \%$. Groups were compared statistically according to NLR. Results: Of the patients, 71 (79.8\%) were male. The patients' mean ages and neutrophil and lymphocyte counts were $67.36 \pm 8.64$ years, $6.89 \pm 3.02 \mathrm{~K} / \mu \mathrm{L}$, and $3.08 \pm 2.18 \mathrm{~K} / \mu \mathrm{L}$, respectively. NLRs of groups 1 and 2 were $2.80 \pm 2.25$ and $4.59 \pm 2.97$, respectively. The relationship between group 1 and 2 tumors was significant $(p=0.008)$. NLR values were $3.82 \pm 2.49$ and $5.20 \pm 3.25$ in groups $2 A$ and $2 B$, respectively. However, no significant relationship was found between these values $(p=0.235)$.

Conclusion: Although no positive correlation was found between NLR and lymph node density, we think that this inflammation marker is an invaluable parameter to predict lymph node metastasis.
\end{abstract}

Keywords: Lymphocyte, neutrophil, bladder cancer, cystectomy

\section{Introduction}

Bladder cancer is the second most common urogenital cancer after prostate cancer (1). The frequency of bladder cancers is increasing with the growing rates of tobacco use and industrialization in societies (2). A study predicted that there will be 81,400 new cases of bladder cancer and 17,980 deaths by 2020 (3). Painless hematuria is the main symptom of patients with bladder cancer $(1,4)$. Bladder cancer is a highly heterogeneous disease in terms of recurrence rate (50-80\%) and progression rate (5-50\%). In terms of tumor stage, approximately $25 \%$ of cases show muscular invasion. Many authors suggested radical cystectomy and pelvic lymph node dissection as the gold standard diagnostic method (5). However, approximately $50 \%$ of these patients can develop distant metastases even after radical cystectomy (6). In this context, oncologists continue to develop neoadjuvant chemotherapy strategies to achieve better clinical outcomes after surgery. These protocols aim to prolong disease-free survival in patients with bladder cancer. However, these approaches have several negative side effects. Previous studies have reported that approximately $20-30 \%$ of patients who underwent neoadjuvant chemotherapy protocols had errors in the clinical staging of bladder tumors, and there may be a delay in the timing of cystectomy or even mortality due to toxicity associated with drug reactions (7). Generally, prognostic models become extremely important in the management of patients with bladder cancer. Numerous studies have demonstrated the clinical significance of various parameters such as tumor size and stage, histopathology, lymphovascular invasion, hydronephrosis, lymph node metastases, lymph node 
density, comorbidity, clinical experience in surgery, and various molecular markers such as p53, p21, p27, and cyclin E1 in predicting the prognosis of patients with bladder cancer $(5,8)$.

The relationship between inflammation and cancer was firstly revealed in 1863 when Rudolf Virchow detected the presence of leukocytes in tumor cells. Numerous studies have been conducted since then to examine the molecular basis of cancer and inflammation. According to these studies, tumor cells, stromal structures, and inflammatory cells surrounding these cells interact coordinately to form an inflammatory tumor microenvironment. Generally, this microenvironment is extremely labile with high plastic properties. Thus, tumor microenvironment and inflammatory reactions are thought to be associated with all the steps of carcinogenesis $(9,10)$. Inflammatory reactions lead to various changes in routine complete blood count parameters. One of these parameters is reproducible, simple, and low-cost neutrophil-lymphocyte ratio (NLR). During systemic inflammation, the increase in neutrophil count and the decrease in lymphocyte count are expected to increase the NLR (6). Based on the link between cancer and inflammation, we aimed to evaluate the relationship between NLR and lymph node metastasis and lymph node density that are shown as a prognostic marker in patients undergoing radical cystectomy for invasive urothelial bladder cancer.

\section{Materials and Methods}

This retrospective analysis analyzed data of patients who underwent radical cystectomy with a diagnosis of bladder cancer in the Urology Clinic of the Faculty of Medicine, Tokat Gaziosmanpaşa University, between 2011 and 2019. The study only included cases with stage 2 urethral bladder cancer according to the 2002 TNM classification. Exclusion criteria were high-grade stage 1 bladder cancer, patient with BCG recurrence, history of neoadjuvant chemotherapy or radiotherapy, other histological subtypes such as adenocarcinoma, squamous cell carcinoma, and small cell carcinoma. In addition, patients with clinical conditions that would change NLR such as secondary malignancy, rheumatological disease, immune deficiency, acute infective pathology, severe endocrinologic disorder, and antiaggregant or anticoagulant use were not included.

The patients' ages, gender, symptoms, and preoperative hemogram parameters were noted. Hemogram parameters were examined in a biochemistry device with regular maintenance and control (Mindray BC-6800, China). These parameters were obtained from preoperative blood analysis results routinely monitored by the anesthesia clinic. NLR was calculated by dividing the neutrophil count obtained in the complete blood count by the lymphocyte count. All patients underwent extended lymph node dissection (obturator, internal and external iliac nodes, deep obturator, common iliac, presacral, precaval, interaortocaval, and para-aortal nodes up to the inferior mesenteric artery) during radical cystectomy. The number of positive lymph nodes was determined for each patient. According to the lymph node status in the surgical specimen, patients with negative status were determined as group 1 and those with positive status as group 2. In total, 62 and 27 patients comprised groups 1 and 2, respectively. The lymph node density level was determined for each patient in group 2. It was calculated by dividing the number of positive lymph nodes by the number of total lymph nodes. Based on the lymph node density value, group 2 was divided into two subgroups. The cut-off value was $20 \%$ in accordance with the large series of studies conducted in previous years (11). Accordingly, group 2A was determined as $<20 \%$, while group $2 B$ as $\geq 20 \%$. Group $2 A$ included only 12 patients. All groups were analyzed according to NLR. The cut-off value for groups 1 and 2 was determined as 1.63. In the evaluation of the cases in group 2 among themselves, the cut-off value was calculated as 4.33 .

The study was carried out following the principles of the Helsinki Declaration and with the approval of the local ethics committee (Tokat Gaziosmanpaşa University, Ethics Committee, confirmation no. 20-KAEK-153).

\section{Statistical Analysis}

Descriptive analyses were performed to provide information on general characteristics of the study population. Quantitative variables were presented as the mean \pm standard deviation and independent samples t-test was used to compare the means of between groups. Qualitative variables were presented as frequency (percent) and chi-square test was used to compare the percentages between groups. Receiver operating characteristics (ROC) curve analysis was used to determine the cut-off value, and the area under the ROC curve was assessed. Correlation analysis was used for examine the relationship between variables. A p-value $<0.05$ was considered significant. Analyses were performed using commercial software (IBM SPSS Statistics 19, SPSS Inc., IBM Corp., Armonk, NY).

\section{Results}

A total of 89 patients were included in the study, of which $71(79.8 \%)$ were male. A total of 67 (75.3\%) patients had a history of smoking. Their mean age and neutrophil and lymphocyte counts were $67.36 \pm 8.64$ years, $6.89 \pm 3.02 \mathrm{~K} / \mu \mathrm{L}$, and $3.08 \pm 2.18 \mathrm{~K} / \mu \mathrm{L}$, respectively. In this study, $67(75.3 \%)$ patients had symptoms of macroscopic hematuria (Table 1). All patients underwent open radical cystectomy and extended lymph node dissection. Procedures were performed by the same surgical team using three different urinary diversion techniques. Moreover, $79(88.7 \%)$ patients were treated with an ileal conduit, 7 (7.9\%) patients had orthotopic neobladder, and 3 (3.4\%) patients had ureterocutaneostomy. All surgical samples were evaluated separately by two experienced pathologists. Of the 35 lymph nodes, 10 were removed. Group 1 consisted of $62(66.7 \%)$ patients. The dermographic data of the groups were

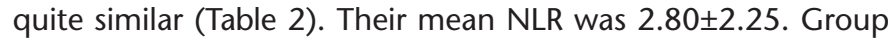
2 had a mean NLR of $4.59 \pm 2.97$. When analyzed with the ROC

\begin{tabular}{|l|l|l|}
\hline Table 1. Signs and symptoms of bladder cancer \\
\hline Signs and symptoms & $\mathbf{n}$ & $\%$ \\
\hline Macroscopic hematuria & 67 & 75.3 \\
\hline Microscopic hematuria & 9 & 10.1 \\
\hline Lower urinary tract symptoms & 8 & 9 \\
\hline Incidental & 5 & 5.6 \\
\hline
\end{tabular}


curve, the cut-off value was 1.63 (Figure 1). Accordingly, we found a significantly higher NLR in group $2(p=0.008)$ (Table 3 ). By contrast, a significant positive and weak relationship was found between NLR and lymph node involvement $(p=0.008)$ (Table 4). Group 2 was further evaluated in two subgroups according to lymph node density. Group 2A was composed of 12 patients. The mean NLR in groups $2 \mathrm{~A}$ and $2 \mathrm{~B}$ were $3.82 \pm 2.49$ and $5.20 \pm 3.25$, respectively. When analyzed with the ROC curve, the cut-off value was 4.33 (Figure 2). Although NLR results were higher in group $2 \mathrm{~B}$ than in group $2 \mathrm{~A}$, a significant relationship could not be established $(p=0.235)$ (Table 5).

\begin{tabular}{|l|l|l|l|}
\hline \multicolumn{2}{|l|}{ Table 2. Comparison of demographic features } & \multirow{2}{*}{ p } \\
\hline \multirow{2}{*}{ Age } & Group & \\
\cline { 2 - 4 } & Group 1 (n=62) & Group 2 (n=27) & \\
\hline Gender (Female/Male) & $67.42 \pm 9.43$ & $67.20 \pm 5.48$ & 0.922 \\
\hline Smoke (Yes/No) & $12 / 50$ & $5 / 21$ & 0.982 \\
\hline $\begin{array}{l}\text { Quantitative datas are shown as mean } \pm \text { standard deviation } \\
\text { Test: Independent Samples t-test and chi-square test } \\
\text { * } p \text {-value less than 0.05 is regarded as statistically significant }\end{array}$ & 0.659 \\
\hline
\end{tabular}

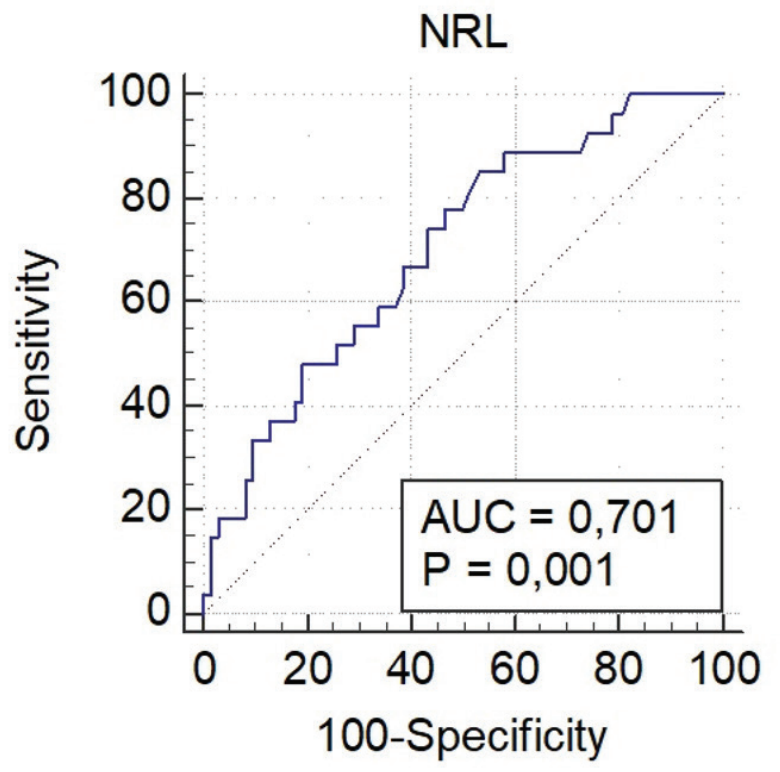

Associated criterion (Cut Off): 1.63

Figure 1. ROC curve for neutrophil-lymphocyte ratio (groups 1-2) ROC: Receiver operating characteristics, AUC: Area under curve

\begin{tabular}{|c|c|c|c|}
\hline & \multicolumn{2}{|l|}{ Group } & \multirow{2}{*}{ p } \\
\hline & Group $1(n=62)$ & Group $2(n=27)$ & \\
\hline NLR & $2.80 \pm 2.25$ & $4.59 \pm 2.97$ & $0.008^{*}$ \\
\hline \multicolumn{4}{|c|}{$\begin{array}{l}\text { NLR: Neutrophil-lymphocyte ratio } \\
\text { Datas are shown as mean } \pm \text { standard deviation } \\
\text { Test: Independent Samples t-test } \\
*^{*} \text {-value less than } 0.05 \text { is regarded as statistically sianificant }\end{array}$} \\
\hline
\end{tabular}

Table 4. Correlation between lymph node involvement and neutrophil-lymphocyte ratio

\begin{tabular}{|l|l|l|}
\hline \multicolumn{2}{|l|}{} & \multicolumn{1}{l|}{$\begin{array}{l}\text { Lymph node } \\
\text { Involvement }\end{array}$} \\
\hline \multirow{2}{*}{ NLR } & $r$ & 0.499 \\
\cline { 2 - 3 } & $\mathrm{p}$ & 0.008 \\
\hline $\begin{array}{l}\text { NLR: Neutrophil-lymphocyte ratio } \\
\text { r: Pearson's correlation coefficient. } \\
\text { A significant positive and weak relationship was found between NLR and lymph } \\
\text { node involvement }\end{array}$ \\
\hline
\end{tabular}

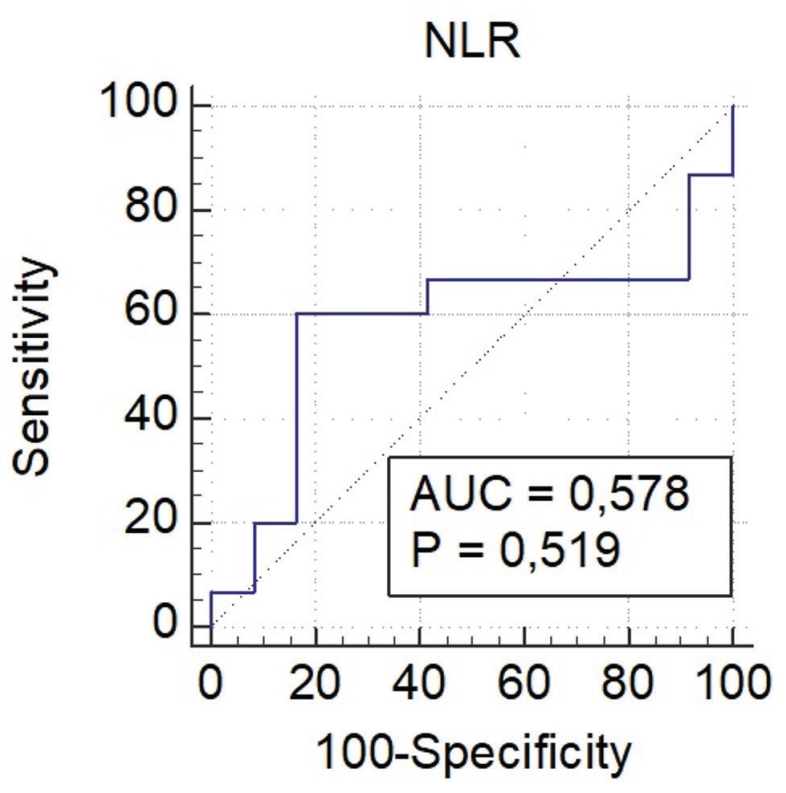

Associated criterion (Cut Off): 4.33

Figure 2. ROC curve for neutrophil-lymphocyte ratio (groups 2A-2B) ROC: Receiver operating characteristics, AUC: Area under curve

\begin{tabular}{|c|c|c|c|}
\hline & \multicolumn{2}{|l|}{ Group } & \multirow{2}{*}{$p$} \\
\hline & Group $2 a(n=12)$ & Group $2 b(n=15)$ & \\
\hline NLR & $3.82 \pm 2.49$ & $5.20 \pm 3.25$ & 0.235 \\
\hline
\end{tabular}

\section{Discussion}

Radical cystectomy has a critical place in the treatment of patients with invasive bladder cancer. However, its clinical results have yet reached the targeted levels (12). In a multicenter study, Shariat et al. (13) evaluated the results of 888 patients who underwent radical cystectomy and reported 5-year mean nonrecurrent and bladder cancer-specific survival rates of $58 \%$ and $66 \%$, respectively. Recently, Stein et al. (14) reported a 5-year recurrence-free and overall survival rates of $68 \%$ and $66 \%$, and a 10 -year recurrence-free and overall survival rates of $60 \%$ and $43 \%$ in their extensive series involving 1.054 patients. The tumor stage is directly related to the heterogeneous biological characteristics of the tumor and still cannot provide clinicians with the desired level of information regarding cancer-specific 
survival in cases of invasive bladder cancer. Determining survival rates and predicting it before surgery is critical in deciding whether to provide treatments in addition to cystectomy such as neoadjuvant chemotherapy. Many models have been developed in this context, and detailed studies are still underway. One of these models is based on lymph node dissection, which is now considered the most important step in radical cystectomy (15). Numerous studies have reported that, on average, one in four patients who underwent radical cystectomy had lymph node involvement (16). In this context, research on the effect of lymph node involvement and its degree on survival have gained great importance in our century. Hautmann et al. (17) reported 10-year recurrence-free and overall survival rates of $59.1 \%$ and $44.9 \%$, respectively, in their study of 788 cases where they discussed the clinical course of the tumor after radical cystectomy. However, analysis of 142 patients with lymph node involvement obtained the same values of $20.9 \%$ and $14.6 \%$, respectively. In a similar study, the results of 336 patients undergoing radical cystectomy and extended pelvic lymphadenectomy were analyzed and 5-year overall and recurrence-free survival rates were $68 \%$ and $69 \%$, respectively. The same study found that $19 \%$ of the patients had lymph node metastases, and lymph node involvement proved a significant adverse prognostic factor with a 5-year probability of survival of $39 \%$ and $76 \%$ (18). Ho et al. (19) evaluated the clinical course of patients after radical cystectomy and reported recurrence-free survival and overall survival rates of $61 \%$ and $53 \%$, respectively, and finally concluded that the number of positive lymph nodes was significantly associated with survival.

Following the acceptance of the effects of lymph node involvement on survival after radical cystectomy by many authors, the scientific world has started to investigate the clinical importance of lymph node density since the beginning of this century. The lymph node density is calculated by dividing the number of density-positive lymph nodes by the number of removed lymph nodes. Herr (11) examined 711 patients with invasive transitional cell carcinoma of the bladder and reported that 162 of his patients had a positive lymph node and an average of 13.3 lymph nodes was removed. His cut-off value for lymph node density was $20 \%$, and he reported a 5-year survival rate of $64 \%$ in cases below the cut-off value and $8 \%$ in those with a cut-off value $>20 \%$. In the same period, Stein et al. (20) examined 1.054 patients who underwent radical cystectomy and lymphadenectomy and reported that $23 \%$ of patients had lymph node metastases, with 5- and 10-year overall recurrencefree survival rates of $35 \%$ and $34 \%$, respectively. Similarly, the lymph node density was analyzed, and the cut-off value was $20 \%$. Moreover, when the lymph node density was $\leq 20 \%$, the 10 -year recurrence-free survival rate was $43 \%$, while it was only $17 \%$ in patients with lymph node density $>20 \%$. Similarly, Wiesner et al. (1) reported 1- and 3-year cancer-specific survival rates of $76 \%$ and $23 \%$ in their study of 152 bladder tumor cases. There was lymph node metastasis in $30 \%$ of the cases. The average lymph node density was 0.11 , and they concluded lymph node density is an independent predictor of cancerspecific survival.

After understanding the importance of lymph node involvement and lymph node density in the clinical course of the tumor following radical cystectomy, clinicians started to investigate how these parameters could be predicted preoperatively. However, a limited number of studies have been conducted on this subject and showed the basis of the relationship between cancer and inflammation (21). Scientists began to understand this relationship when the presence of leukocytes in tumor cells was observed about 1.5 centuries ago. The link between inflammation and cancer has become clearer with the introduction of many molecular-based new technologies in medicine. Infection and inflammation are held responsible for nearly a quarter of cancer cases today $(9,10)$. Macrophages and T-cells are active immune cells around the tumor microenvironment. Increased cytokine and chemokine expression are directly related to the specificity of these cells. This medium triggers tumor growth, angiogenesis, invasion, and metastasis. Many cytokines such as interleukin (IL)-1, IL-6, IL-17, and IL-23 have an extremely important place in cancer progression. In addition, tumor necrosis factor-alpha, transforming growth factor-beta, FasL, and estimated glomerular filtration rate ligands contribute to the development and survival of tumor cells. Reactive oxygen species also play a role in a different pathway. These molecules can trigger cancer formation by neutralizing important tumor suppressor genes such as Tgfbr2 and Bax. Similarly, irreversible protein modification is caused by oxidative stress that causes critical damage to the cellular genome. All these vital steps affecting cariogenesis constitute the molecular basis of many types of cancer, for example, several clinical conditions such as liver or stomach cancers that develop due to viral effects, colorectal cancers triggered by inflammatory bowel disease, and lung cancer secondary to chronic inflammation caused by environmental factors such as smoking $(9,22)$.

This relationship between cancer and inflammation has been investigated in detail in patients undergoing radical cystectomy for bladder tumors. Gongo et al. (6) examined 189 patients with bladder cancer and reported that NLR was correlated with the clinical results of radical cystectomy for the first time. They concluded that tumor size, clinical T-stage, hydronephrosis, concomitance of carcinoma in situ, and NLR were significantly associated with poor prognosis. In another study, Kawahara et al. (23) evaluated 74 patients undergoing radical cystectomy and stated that the cut-off value for NLR was 2.38, and this parameter had predictive value in predicting the prognosis. In a similar study of 84 cases, Tan et al. (24) reported 2.7 as the cutoff value for NLR and considered high NLR to be independently associated with higher recurrence rate, higher T-staging, and lymph node involvement. In their large series of 385 cases, Kang et al. (25) reported 2 as the cut-off value of NLR and that high NLR was directly related to advanced tumor stage, lymphovascular invasion, and lymph node involvement. In the same study, cancer-specific survival and overall survival rates were poor in cases with high NLR. In another large-series study, Yoshida et al. (26) examined 323 patients with bladder cancer who underwent radical cystectomy and reported that both preand postoperative NLR were related to overall survival. However, Ojerholm et al. (27) suggested that NLR had no predictive value for overall survival in patients with muscle-invasive bladder cancer in their large-scale studies. Likewise, Tang et al. (28) reported 
the lack of a relationship between NLR and tumor stage in their study of 302 patients with bladder cancer. Moreover, Demirtaş et al. (21) examined 201 cases and suggested that preoperative NLR was not correlated with lymph node density. In the present study, a significant relationship was found between NLR and lymph node involvement. However, NLR increased in the group with high lymph node density, albeit not significant.

\section{Study Limitations}

As limitations, this study is a single-center retrospective study with a limited number of patients. Moreover, this study lacks long-term follow-up results such as disease-free survival and overall survival, which is another deficiency of our clinical data. However, in this study, the lymph node status, which is very important in clinical follow-up after cystectomy, was studied in detail.

In this study, lymph node involvement and lymph density are directly related to prognosis in patients with bladder cancer as reported in many studies. The clinical importance of neoadjuvant chemotherapy in patients with bladder cancer is currently accepted by nearly all authors of tumors. Moreover, it is critical to consider the side effects of current treatment strategies that can result in serious mortality and morbidity. In this context, clinicians should select the ideal patient by managing the benefit-loss equation correctly.

\section{Conclusion}

NLR evaluated before radical cystectomy in patients with stage 2 ureteral bladder cancer is extremely useful in predicting lymph node involvement with high prognostic significance after surgery. However, we believe that our results should be supported by large-scale, randomized, multicenter studies.

\section{Acknowledgements}

Publication: The results of the study were not published in full or in part in form of abstracts.

Contribution: There is not any contributors who may not be listed as authors.

Conflict of Interest: No conflict of interest was declared by the authors.

Financial Disclosure: The authors declared that this study received no financial support.

\section{Ethics}

Ethics Committee Approval: The study was carried out following the principles of the Helsinki Declaration and with the approval of the local ethics committee (Tokat Gaziosmanpasa University, Ethics Committee, confirmation no. 20-KAEK-153).

Informed Consent: Retrospective study.

Peer-review: Externally peer-reviewed.

\section{Authorship Contributions}

Concept: E.K., D.A., Y.E.K., Design: E.K., D.A., Y.E.K., L.M.Ö., V.U., Data Collection or Processing: D.A., Y.E.K., Analysis or
Interpretation: L.M.Ö., V.U., Literature Search: E.K., L.M.Ö., V.U., Writing: E.K.

\section{References}

1. Wiesner C, Salzer A, Thomas C, et al. Cancer-specific survival after radical cystectomy and standardized extended lymphadenectomy for node-positive bladder cancer: prediction by lymph node positivity and density. BJU Int 2009;104:331-335.

2. Degeorge KC, Holt HR, Hodges SC. Bladder Cancer: diagnosis and treatment. Am Fam Physician 2017; 96:507-515.

3. Siegel RL, Miller KD, Jemal A. Cancer statistics, 2020. CA Cancer J Clin 2020;70:7-30.

4. Yaxley JP. Urinary tract cancers: an overview for general practice. Family Med Prim Care 2016;5:533-538.

5. Ataus S. Yüksek riskli kas invaziv olmayan mesane kanserlerinde yaklaşım ve tedavi Bull Urooncol 2007;6:15-20.

6. Gondo T, Nakashima J, Ohno Y, et al. Prognostic value of neutrophilto-lymphocyte ratio and establishment of novel preoperative risk stratification model in bladder cancer patients treated with radical cystectomy. Urology 2012;7:1085-1091.

7. Baltacı $S$. Mesane tümörlerinde neoadjuvan ve adjuvan kemoterapi. Bull Urooncol 2007;6:3-8.

8. Türkölmez K. Radikal sistektomi öncesi ve sonrası nomogram kullanımı: Yakın gelecekteki rolü. Bull Urooncol 2009;8:21-26.

9. Yıldırım I. The role of inflammation in cancer development. Eskişehir Technical University Journal of Science and Technology C- Life Sciences and Biotechnology 2019;8:121-140.

10. Greten FR, Grivennikov SI. Inflammation and cancer: triggers, mechanisms, and consequences. Immunity 2019;51:27-41.

11. Herr HW. Superiority of ratio based lymph node staging for bladder Cancer. J Urol 2003;169:943-945.

12. Tekin A. Radikal sistektomi sonrası nükslerin tanı ve tedavisi. Bull Urooncol 2008;7:10-17.

13. Shariat SF, Karakiewicz PI, Palapattu GS, et al. Outcomes of radical cystectomy for transitional cell carcinoma of the bladder: a contemporary series from the bladder cancer research consortium. J Urol 2006;176:2414-2422.

14. Stein JP, Lieskovsky G, Cote R, et al. Radical cystectomy in the treatment of invasive bladder cancer: long-term results in 1,054 patients. J Clin Oncol 2001;19:666-675.

15. Skinner DG. Management of invasive bladder cancer: a meticulous pelvic node dissection can make a difference. J Urol 1982;128:34-36.

16. $\mathrm{Ku} \mathrm{JH}$, Kang M, Kim HS, et al. Lymph node density as a prognostic variable in node-positive bladder cancer: a meta-analysis. BMC Cancer 2015; 15:447.

17. Hautmann RE, Gschwend JE, de Petriconi RC, et al. Cystectomy for transitional cell carcinoma of the bladder: results of a surgery only series in the neobladder era. J Urol 2006;176:486-492.

18. Steven K, Poulsen A. Radical cystectomy and extended pelvic lymphadenectomy: survival of patients with lymph node metastasis above the bifurcation of the common lliac vessels treated with surgery only. J Urol 2007;178:1218-1223.

19. Ho CH, Huang CY, Lin WC, et al. Radical cystectomy in the treatment of bladder cancer: oncological outcome and survival predictors. I Formos Med Assoc 2009;108:872-878.

20. Stein JP, Cai J, Groshen S, Skinner DG. Risk factors for patients with pelvic lymph node metastases following radical cystectomy with en bloc pelvic lymphadenectomy: the concept of lymph node density. J Urol 2003;170:35-41.

21. Demirtaş A, Sabur V, Akınsal EC, et al. Can neutrophil-lymphocyte ratio and lymph node density be used as prognostic factors in patients undergoing radical cystectomy? ScientificWorldjournal 2013;2013:703579. doi: 10.1155/2013/703579. 
22. Hussain SP, Harris CC. Inflammation and cancer: an ancient link with novelpotentials. Int J Cancer 2007; 121:2373-2380.

23. Kawahara T, Furuya K, Nakamura M, et al. Neutrophil-to-lymphocyte ratio is a prognostic marker in bladder cancer patients after radical cystectomy. BMC Cancer 2016;16:185.

24. Tan YG, Eu E, Lau Kam On W, Huang HH. Pretreatment neutrophilto-lymphocyte ratio predicts worse survival outcomes and advanced tumor staging in patients undergoing radical cystectomy for bladder cancer. Asian J Urol 2017;4:239-246.

25. Kang M, Jeong CW, Kwak C, et al. the prognostic significance of the early postoperative neutrophil-to-lymphocyte ratio in patients with urothelial carcinoma of the bladder undergoing radical cystectomy. Ann Surg Oncol 2016;23:335-342.

26. Yoshida T, Kinoshita H, Yoshida K, et al. Perioperative change in neutrophil-lymphocyte ratio predicts the overall survival of patients with bladder cancer undergoing radical cystectomy. Jpn J Clin Oncol 2016;46:1162-1167.

27. Ojerholm E, Smith A, Hwang WT, et al. Neutrophil-to-lymphocyte ratio as a bladder cancer biomarker: Assessing prognostic and predictive value in SWOG 8710. Cancer 2017;123:3856.

28. Tang X, Wang S, An C, et al. Preoperative high neutrophil-tolymphocyte ratio is associated with high-grade bladder cancer. Anticancer Res 2017;37:4659-4663. 